\title{
Plans d'expérience et analyse des corrélations pour la résolution du problème de formation de cellules avec gammes alternatives
}

\author{
Wafik Hachicha ${ }^{1, a}$, Faouzi Masmoudi ${ }^{2}$ et Mohamed HaddaR ${ }^{2}$ \\ 1 Unité de recherche de Mécanique, Modélisation et Production (U2MP), École Nationale d'ingénieurs de Sfax, B.P.1173, \\ 3038 Sfax, Tunisie, Institut supérieur de Gestion Industrielle de Sfax, Tunisie \\ 2 Unité de recherche de Mécanique, Modélisation et Production (U2MP), Département de Génie Mécanique, École \\ Nationale d'ingénieurs de Sfax, B.P.1173, 3038 Sfax, Tunisie
}

Reçu le 4 avril 2008, accepté le 12 mai 2009

\begin{abstract}
Résumé - La formation des cellules (FC) de production se traduit par le groupement des produits en familles et le partage des machines en sous-groupes. Le traitement de ce problème devient plus complexe en présence de gammes alternatives. Dans ce contexte, nous proposons une nouvelle approche qui traite ce problème en deux niveaux. Le premier niveau porte sur l'application de la méthode des plans d'expériences, l'objectif étant de choisir, pour chaque produit, la gamme préférentielle permettant l'optimisation des indicateurs de performance de FC. En plus, ce niveau de traitement permet une analyse de la sensibilité de la solution finale ignorée par les techniques antérieures de la FC. Nous avons proposé et justifié un nouvel indice de la FC défini par le pourcentage de conservation des données de la matrice d'incidence initiale. Le second niveau de traitement consiste à appliquer la méthode de la FC basée sur l'analyse des corrélations statistiques entre les différents produits et machines. Pour expliquer l'approche proposée, nous avons détaillé deux exemples numériques. Les résultats d'une analyse comparative appliquée à des problèmes reconnus en littérature, ont permis de valider l'approche proposée.
\end{abstract}

Mots clés : Formation des cellules / gammes alternatives / analyse des corrélations / plan d'expériences / analyse de sensibilité / indice de performance

\begin{abstract}
Design of experiment and correlation analysis for solving cell formation problem with alternative routings. Cell formation (CF) problem involves grouping parts into families and machines into cells. The CF problem becomes extra complicated if parts are assigned with alternative routings. In this context, we propose a new two-phase approach. The first phase is based on Designed of Experiments. The objective is to realize the best alternative routing for each part in term of CF performance criteria. In addition, the result goes across and beyond the previous CF procedures by providing a sensitivity analysis aspect. A new performance criterion which is based on the percentage of conservation of incidence matrix data is proposed and validated. The second phase consists in applying CF based on correlation analysis to cluster the machines and parts. In order to illustrate the two phases of the proposed approach, two numerical examples are provided. Furthermore, the results of a comparative study based on multiple performance criteria show that the present approach is valid.
\end{abstract}

Key words: Cell formation / alternative routings / correlation analysis / design of experiments / sensitivity analysis / performance criteria

\section{Introduction}

Le problème de formation des cellules (FC) est défini comme une application classique de la Technologie de Groupe [1]. Dans ce cas la production cellulaire consiste à fabriquer des produits similaires (famille de produits)

\footnotetext{
a Auteur pour correspondance :

wafik_hachicha@yahoo.fr
}

au moyen d'un ensemble de machines non similaires partitionnées en cellules [2]. Le problème est donc de déterminer ces familles de produits et les groupes de machines correspondantes. Le groupement des produits en familles se fait en se basant sur la similarité de ces produits : mêmes composantes, mêmes opérations, etc. Les machines peuvent être groupées en fonction des gammes opératoires dans l'optique de limiter les 
mouvements intercellulaires et de réduire les manutentions. Burgess et al. [3] affirment qu'avec l'aménagement cellulaire, les temps de transfert, les temps de cycle et les en-cours seront réduits par rapport aux autres types d'aménagements.

Souvent, les méthodes de FC traitent des problèmes en supposant que chaque produit est défini par une gamme unique. En réalité chaque produit peut être traité suivant plusieurs scénarios de fabrication (gammes alternatives). Le problème de formation de cellules à gamme alternatives (FCGA) devient plus complexe car il sera nécessaire d'une part de sélectionner une seule gamme par produit et d'autre part de faire le groupement des cellules. De nombreux auteurs proposent différentes approches pour la résolution du problème de FCGA [4-10].

Adenso-Diaz et al. [11] proposent une classification des méthodes de résolution du FCGA en trois catégories principales : méthodes séquentielles, méthodes itératives et méthodes simultanées. Dans [7,12] les auteurs ont proposé des méthodes de la première catégorie en deux étapes. Pendant la première étape, une gamme est déterminée définitivement pour chaque produit en respectant les contraintes de capacité des machines ainsi que les familles de produits. Dans la deuxième étape, la mise en cellules est effectuée.

Nagi et al. [5] proposent une méthode itérative résolvant deux sous-problèmes distincts : le premier sous problème de la FCGA à l'aide d'une heuristique. Le second sous-problème concerne la sélection des gammes par l'algorithme de Simplex. Cette méthode est limitée car le nombre de contraintes devient très important quand la taille des problèmes augmente. De plus, l'utilisation d'une heuristique ne peut pas garantir la convergence vers un optimum global.

D'autres auteurs ont proposé des méthodes de résolution simultanée pour la FCGA. Divers techniques sont ainsi proposées. Caux et al. [13] ont combiné une approche basée sur la technique de recuit simulé et sur l'algorithme de Séparation et Evaluation «branchand-bound » pour améliorer simultanément la sélection des gammes et la minimisation du flux intercellulaire. Adenso-Diaz et al. [11] ont proposé des algorithmes basés sur la technique de type recherche Tabou. Vin et al. [14] et Chan et al. [10] ont proposé divers algorithmes génétiques pour la résolution de ce problème.

Comme indiqué par la plupart des auteurs, les différentes méthodes itératives ou simultanées, ne sont pas pratiques pour résoudre des problèmes de FCGA de grandes tailles. En plus, une fois la solution optimale trouvée, les données des différentes gammes possibles ne seront pas exploitables ultérieurement. Pour résoudre ce problème d'une manière efficace, nous suggérons une approche séquentielle modifiée comme recommandé par Gangan et al. [15], Hwang et Ree [16], Kazerooni et al. [17], Kizil et Ozbayrak [18], etc. Dans cette catégorie d'approches, non indiquée par Adenso-Diaz et al. [11], le problème de FCGA est subdivisé en deux sousproblèmes en cascade. Le premier sous-problème porte sur la sélection optimale d'une seule gamme préférentielle

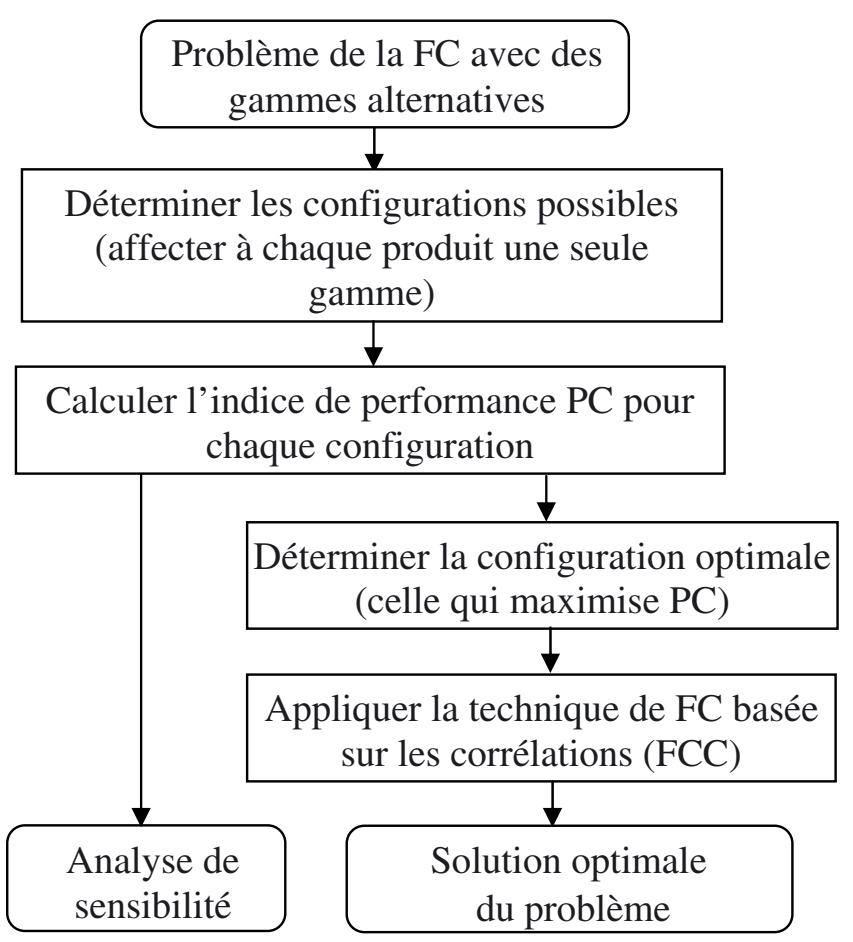

Fig. 1. Architecture de l'approche proposée.

pour chaque produit et qui assure une meilleure mise en cellules ultérieurement. Le second sous problème consiste à appliquer une des méthodes de FC.

C'est dans cette optique que s'inscrit ce présent travail. Il s'agit d'exploiter les gammes possibles de chaque produit non seulement durant la conception des cellules mais aussi à l'analyse de sensibilité pour une meilleure exploitation du système de production cellulaire. L'approche que nous proposons porte :

- en premier lieu, sur le choix de la gamme préférentielle pour chaque produit en se basant sur la technique des plans d'expériences. Ceci nous a permis d'introduire une analyse de sensibilité de la solution optimale selon les différentes gammes possibles;

- en second lieu, sur le groupement des produits en familles et des machines en cellules qui se base sur la méthode de corrélation statistique.

Une description détaillée de l'approche proposée ainsi que la définition et la justification de l'indice de performance proposé sont présentées au paragraphe 2 . Le paragraphe 3 présente le traitement de deux exemples relatifs à des problèmes reconnus en littérature. Le paragraphe 4 indique les résultats de l'étude comparative réalisée pour valider l'approche proposée. Les conclusions et les perspectives de cette étude sont présentées au paragraphe 5.

\section{Approche proposée}

\subsection{Architecture de l'approche proposée}

La démarche de l'approche proposée est illustrée dans la figure 1 en deux phases. 
La première phase porte sur l'application de la méthode de plan d'expériences. En effet, chaque expérience constitue une solution possible du problème de la FCGA. La réponse du modèle du plan d'expérience adoptée est le pourcentage cumulé (PC) qui permet la mesure d'une solution de la FC. La seconde phase de la démarche proposée consiste à compléter la résolution du FCGA en se basant sur la FC de la solution optimale trouvée en première phase. La démarche de l'approche proposée ainsi que le critère PC seront détaillés dans les paragraphes suivants.

\subsection{Formation des cellules basée sur les corrélations}

Arvindh et Irani [19] sont les premiers à utiliser la méthode d'Analyse en Composantes Principales (ACP) pour étudier la faisabilité de FC. Ils proposent d'utiliser cette méthode comme une première étape pour le classement de plusieurs produits en familles. La seconde étape consiste à appliquer l'une des méthodes de diagonalisation de matrice par blocs pour résoudre le problème de FC. Albadawi et al. [20] ont proposé une démarche en deux étapes. La première consiste à utiliser l'ACP en se basant sur l'indice de similarité de Jaccard, pour la formation des groupes de machines seulement. Dans une seconde étape, ils ont proposé un algorithme pour l'affectation des produits aux cellules ainsi formées. Dans ce travail, nous avons utilisé la nouvelle définition du coefficient de similarité proposé par Hachicha et al. [21] qui nous a permis d'appliquer la méthode ACP d'une autre manière que dans Arvindh et Irani [19] et Albadawi et al. [20]. L'intérêt consiste à obtenir les familles de produits et les sous-groupes de machines simultanément. Dans la suite de l'article, cette méthode sera appelée FCC (formation des cellules basée sur les corrélations).

La méthode FCC passe par deux étapes. Dans une première étape, la matrice de corrélation est utilisée comme une matrice de similarité entre les produits et les machines. Dans une seconde étape, l'application de la méthode (ACP) et l'analyse graphique du plan principal permettent d'une part, le partage des machines en groupes et le groupement des produits en familles et d'autre part, l'identification des machines et des produits exceptionnels.

\subsubsection{Matrice des corrélations}

Soit un atelier possédant $« m »$ machines et $« p »$ produits. Soit $A$, la matrice d'incidence binaire du problème. Cette matrice $p \times m$ est représentée dans l'équation (1).

$$
A=\left(\begin{array}{cccc}
a_{11} & a_{12} & \ldots & a_{1 m} \\
a_{21} & a_{22} & & a_{2 m} \\
\cdot & & & \\
\cdot & & & \\
\cdot & & & \\
a_{p 1} & a_{p 2} & & a_{p m}
\end{array}\right)
$$

Avec $a_{i j}=1$ si la machine $j$ traite le produit $i, a_{i j}=0$ sinon

Soit $M_{j}$ le vecteur machine déduit de la matrice d'incidence $A: M_{j}^{\mathrm{A}}\left[a_{1 j}, a_{2 j}, \ldots, a_{p j}\right]$.

La matrice d'incidence obtenue présente, surtout pour les problèmes de grandes tailles, une masse d'informations difficilement accessibles. L'approche de la FC se base sur l'analyse de cette matrice, pour savoir et expliquer la liaison éventuelle qui existe entre les différents machines et produits. Afin de rendre le résultat indépendant des charges affectées à chaque machine, un prétraitement indispensable consiste à centrer et réduire les vecteurs machines. Cette opération permet de mieux voir les différences relatives. Les centres de gravité des machines sont alors confondus à l'origine. On note par $B$ la matrice d'incidence traitée de $A$.

$$
M_{j}^{B}=\frac{M_{j}^{A}-E_{j}}{\sigma_{j}}
$$

Avec $E_{j}$ est la moyenne et $\sigma_{j}$ est l'écart type des éléments du vecteur machine $M_{j}$.

Les expressions de $E_{j}$ et de $\sigma_{j}$ sont présentées respectivement dans les équations (3) et (4).

$$
E_{j}=\frac{\sum_{k=1}^{p} a_{k j}}{p}
$$

et

$$
\sigma_{j}^{2}=\frac{1}{p} \sum_{k=1}^{p}\left(a_{k j}-E_{j}\right)^{2}
$$

La matrice des corrélations est définie par

$$
S=\frac{1}{p} B^{t} B
$$

Où $B^{t}$ désigne la matrice transposée de $B$.

\subsubsection{Application de la méthode ACP}

La seconde étape de la méthode FCC consiste à appliquer la méthode ACP à la matrice d'incidence traitée B. Les produits sont alors considérés comme les « individus » et les machines sont vues comme les «variables». Cette méthode se base sur la recherche des valeurs propres et des vecteurs propres associés à la matrice des corrélations $S$. La matrice $S$ est symétrique, définie positive, elle admet d'avoir $m$ valeurs propres réelles positives. Ces valeurs propres, (notées $\lambda_{i}$ ) sont les solutions de l'équation (6).

$$
\operatorname{det}\left(S-\lambda_{i} I\right)=0 ; \quad i \in\langle 1, m\rangle
$$

Classons les $\lambda_{i}$ par ordre croissant : $\lambda_{1} \geq \lambda_{2} \geq \ldots \geq$ $\lambda_{m}$. Pour chaque vecteur propre $\lambda_{i}$ est associé un vecteur propre noté $C_{i}$, sachant que I désigne la matrice identité de dimension $m$.

Par la suite, la méthode FCC consiste à projeter les points «machines » et les points « produits » dans le plan 


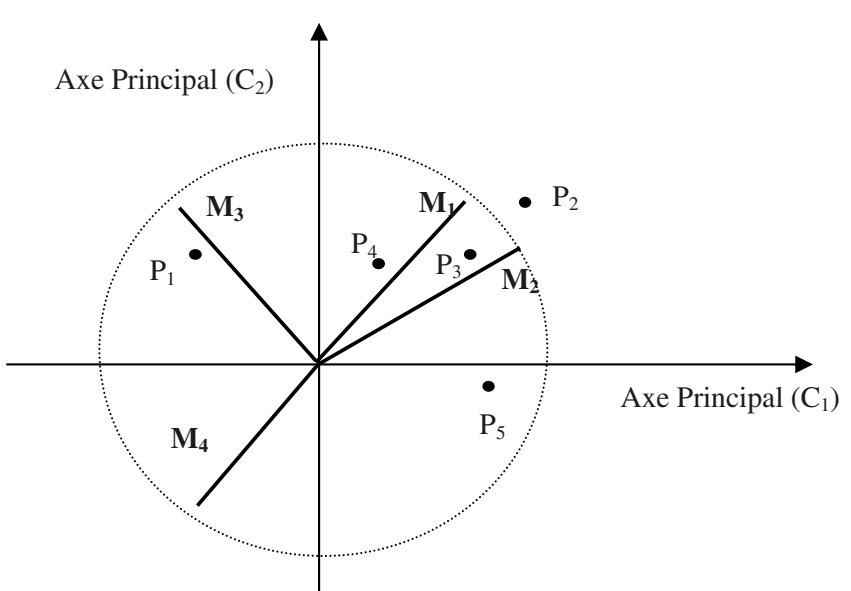

Fig. 2. Un exemple d'illustration du plan principal.

principal. Un exemple de projection sur un plan principal est donné dans la figure 2. Le plan principal est formé par les premiers axes principaux de vecteurs unitaires respectifs $\left(C_{1}, C_{2}\right)$.

Les machines forment un cercle centré sur l'origine. Le rayon de ce cercle (où se trouvent les machines) est totalement arbitraire et ne présente pas une signification particulière. En revanche, la direction de la machine (vecteur machine) joignant l'origine à ce point est très significative en termes de corrélation. Leurs positions respectives donnent le niveau de corrélations. En effet, le coefficient de corrélation de deux machines est le carré du cosinus de l'angle qui joint ces deux machines.

L'analyse du plan principal, pour le groupement des machines en cellules, permet de constater qu'il existe les trois situations typiques suivantes :

- deux machines voisines sont fortement corrélées. Ceci explique leurs appartenances à une même cellule. C'est le cas des machines $\left(\mathrm{M}_{1}\right.$ et $\left.\mathrm{M}_{2}\right)$ de l'exemple présenté sur la figure 2 ;

- deux machines trouvées pratiquement opposées $(\pi)$ sont aussi très corrélées, mais négativement. Ils ne doivent pas appartenir à une même cellule. C'est le cas des machines $\left(\mathrm{M}_{1}\right.$ et $\left.\mathrm{M}_{4}\right)$ qui ne doivent pas être regroupées pour former la même cellule;

- deux machines trouvées à angle approximativement droit $(\pi / 2)$, sont classées comme des machines indépendantes dont la corrélation est nulle. C'est le cas des machines $\left(M_{1}\right.$ et $\left.M_{3}\right)$ et $\left(M_{3}\right.$ et $\left.M_{4}\right)$ qui ne doivent pas être regroupées dans la même cellule.

De la même manière, on regroupe les produits en familles. Dans ce cas, on peut présenter quelques règles de groupage des produits et cela pour les situations typiques suivantes :

- deux ou plusieurs produits sont positionnés pour former un ensemble de points très proches les uns des autres. Ceci explique que ces produits ont presque la même gamme de production et doivent appartenir à la même famille de produits. Les produits $\left(\mathrm{P}_{2}, \mathrm{P}_{3}\right.$ et $\left.\mathrm{P}_{4}\right)$ de l'exemple développé représente un cas type de la formation de famille des produits;
- un produit est très proche de l'axe orienté positivement d'une machine. Ceci explique sa nécessité pour cette machine. Le produit sera affecté à la cellule qui compose la machine correspondante. C'est le cas ici, par exemple pour les couples $\left(\mathrm{P}_{4}\right.$ et $\left.\mathrm{M}_{1}\right)$ et pour $\left(\mathrm{P}_{1}\right.$ et $\left.\mathrm{M}_{3}\right)$.

Pour plus de détails sur la démarche et les avantages de la méthode FCC, nous invitons le lecteur à consulter [21] pour des matrices d'incidences binaires, et [22] pour des matrices d'incidences à durées opératoires.

\subsection{Plan d'expériences}

Pour la recherche de la solution optimale ainsi que l'analyse de sensibilité, nous avons utilisé la méthode des plans d'expériences. En effet, cette méthode possède plusieurs avantages par rapport aux méthodes classiques d'expérimentation : diminution du nombre d'essais accomplis, détection des éventuelles interactions entre facteurs, modélisation aisée des problèmes et obtention des résultats avec une bonne précision. Ces avantages contribuent au développement actuel de cette méthode dans plusieurs secteurs d'activité [23]. L'analyse de sensibilité permet d'estimer l'évolution des indices de sorties d'un système en fonction des modifications apportées aux paramètres d'entrées [24]. Cela revient à étudier le comportement des entrées et des sorties du système dans un espace expérimental.

Un plan d'expériences est un tableau ou une matrice dont les colonnes représentent des facteurs et les lignes des essais. La dernière colonne représente la réponse mesurée du système en se fixant un seul niveau de chaque facteur étudié. Il existe principalement deux types de plans d'expériences : plan factoriel et plan fractionnaire.

Plan factoriel : ils contiennent toutes les combinaisons des facteurs dans tous les niveaux possibles. Le nombre d'essais à réaliser devient rapidement non maîtrisable c'est-à-dire qu'il correspond au produit du nombre de niveaux de chaque facteur. C'est l'inconvénient majeur qui justifie la théorie de construction des plans.

Plan fractionnaire : c'est une fraction du plan complet. Le nombre d'essais est réduit par rapport au plan complet. En contrepartie il ne permet d'analyser qu'un sous-ensemble du modèle complet car des effets peuvent être mélangés. Les plans d'expériences de Taguchi font partie de ces plans fractionnaires, mais avec un avantage important, la facilité de mise en pratique de ces plans grâce à quelques astuces qui simplifient la tâche de l'expérimentateur.

Concernant le choix d'une table de Taguchi, il faut calculer le nombre d'essais minimum à effectuer. Ce calcul est basé sur la notion de degré de liberté (ddl) qui est défini mathématiquement comme suit :

- le ddl d'un facteur est égal au nombre de ses niveaux moins un;

- le ddl d'une interaction est égal au produit des ddl des facteurs la composant; 


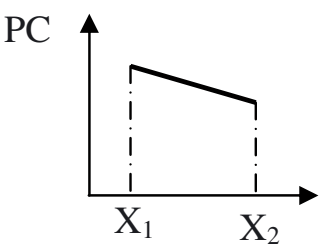

(a)

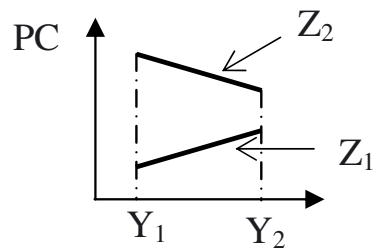

(b)
Fig. 3. Graphes de l'évolution des effets.

- le ddl d'un modèle est égal à la somme des ddl des facteurs et des interactions ;

- le ddl d'un plan est égal au nombre d'essais moins un.

Il s'agit alors de choisir la plus petite matrice d'expériences compatible avec le modèle choisi, c'est-àdire le nombre de ddl du plan doit être supérieur ou égal à celui du modèle. Les tableaux de Taguchi sont désignés par trois symboles. Par exemple, la table $\mathrm{L}_{8}\left(2^{7}\right)$ comporte 8 essais pour étudier un problème possédant au maximum 7 facteurs avec deux niveaux chacun. La table $\mathrm{L}_{36}$ $\left(2^{11} \times 3^{12}\right)$ comporte 36 essais pour étudier 11 facteurs à deux niveaux et 12 autres facteurs à trois niveaux.

Dans l'approche proposée, les entrées constituent les différentes configurations possibles (choisir une seule gamme possible pour chaque produit) et les sorties sont les PC de chaque configuration possible. L'indicateur PC constitue le pourcentage d'inertie expliqué par le plan principal (le pourcentage de conservation des données de la matrice d'incidence). Il peut être défini par les deux premiers valeurs propres : $\lambda_{1}$ et $\lambda_{2}$. Par définition l'indice $\mathrm{PC}$ est inférieur ou égal à $100 \%$.

$$
P C=\frac{\lambda_{1}+\lambda_{2}}{\sum_{k=1}^{m} \lambda_{k}}=\frac{\lambda_{1}+\lambda_{2}}{m}
$$

La technique que nous proposons, pour l'analyse de l'incidence liée à la variation des gammes, consiste à mesurer les $\mathrm{PC}$ en modifiant, à chaque fois, une seule gamme de l'ensemble des produits à traiter. Ensuite, l'évolution des résultats liés à chacun des produits est présentée par des graphes. Par exemple, la figure 3 illustre les effets des produits $\mathrm{X}, \mathrm{Y}$, et $\mathrm{Z}$, ayant chacun deux gammes de traitement possibles. Ces gammes sont notées respectivement par: $\mathrm{X}_{1}, \mathrm{X}_{2}, \mathrm{Y}_{1}, \mathrm{Y}_{2}, \mathrm{Z}_{1}$ et $\mathrm{Z}_{2}$.

L'effet moyen d'une gamme est proportionnel à la pente de la droite présentée sur le graphe de la figure $3 a$. La présence d'une interaction sur le graphe de la figure $3 \mathrm{~b}$ se note lorsque les deux droites (segments $Z_{1}$ et $Z_{2}$ ) ne sont pas parallèles.

Dans ce cas présent, supposé à valeurs déterministes, l'analyse de la sensibilité à l'aide d'un plan d'expériences n'exige pas d'étudier statistiquement la solution finale. Par conséquence, la répétition et la randomisation des expériences sont inutiles puisque celles-ci donnent la même réponse pour une même configuration.

\subsection{Les critères de performances}

Plusieurs indices de performances dans le contexte de FC ont été proposés en littérature. L'objectif de ces indices est de mesurer le degré de la mise en cellules et par conséquent de prévoir l'importance des flux entre les cellules formées. La majorité des auteurs utilise ces indices pour comparer l'efficacité des méthodes qu'ils proposent avec les différentes méthodes antérieures [20,25-28]. Ces indices sont relatifs seulement à la phase de conception des aménagements cellulaires et ne permettent pas de mesurer directement le trafic intercellulaire dans la phase exploitation. En effet, la mesure finale des flux entre les cellules dépend de plusieurs paramètres d'exploitation tels que : les demandes des produits, la taille des lots, les temps de réglages des machines, les distances de transfert, etc. [28]. Dans le cadre de ce travail, nous allons comparer et justifier la pertinence de l'indice PC que nous avons proposé par rapport aux autres indices de performance utilisés en littérature.

\subsubsection{Pourcentage des éléments exceptionnels}

Le pourcentage des éléments exceptionnels $(P E)$ est l'indice de performance le plus utilisé en littérature. La qualité d'une méthode de $F C$ est mesurée par le nombre des « $1 »$ trouvés hors blocs diagonaux. Ces éléments sont appelés les éléments exceptionnels.

L'expression de l'indice PE est la suivante :

$$
P E=\frac{E E}{N E} \times 100
$$

Avec :

$N E$ le nombre de « $1 »$ dans la matrice d'incidence. $E E$ est le nombre d'éléments exceptionnels.

\subsubsection{Taux d'utilisation des machines}

Le taux d'utilisation des machines MU est proposé comme un indice de performance par [29]. Il indique l'occupation moyenne des machines dans les cellules. Si ce taux est augmenté, la solution est supposée meilleure.

$$
M U=\frac{N E-E E}{\sum_{k=1}^{Q} m_{k} p_{k}}
$$

avec :

$Q$ le nombre de cellules.

$m_{k}$ est le nombre de machines affecté à la $k^{\text {ième }}$ cellule. $p_{k}$ est le nombre de produits affecté à la $k^{\text {ième }}$ cellule.

\subsubsection{Efficience du groupement}

Il existe deux définitions différentes pour ce critère. La première définition a été proposée par [29]. Elle tient en 
Tableau 1. Les données de l'exemple illustratif.

\begin{tabular}{|c|c|c|c|c|c|c|c|c|c|}
\hline Produit & Gamme & $\mathrm{M}_{1}$ & $\mathrm{M}_{2}$ & $\mathrm{M}_{3}$ & $\mathrm{M}_{4}$ & $\mathrm{M}_{5}$ & $\mathrm{M}_{6}$ & $\mathrm{M}_{7}$ & $\mathrm{M}_{8}$ \\
\hline \multirow{2}{*}{ P1 } & G1 & 0 & 0 & 1 & 0 & 1 & 0 & 1 & 0 \\
\cline { 2 - 11 } & $\mathrm{G} 2$ & 0 & 0 & 1 & 0 & 1 & 1 & 0 & 1 \\
\hline P2 & G1 & 1 & 0 & 1 & 0 & 0 & 0 & 1 & 0 \\
\hline \multirow{3}{*}{ P3 } & G1 & 1 & 0 & 0 & 0 & 1 & 0 & 0 & 1 \\
\cline { 2 - 11 } & G2 & 1 & 0 & 1 & 1 & 0 & 0 & 1 & 0 \\
\cline { 2 - 11 } & G3 & 1 & 1 & 0 & 0 & 1 & 0 & 1 & 1 \\
\hline P4 & G1 & 0 & 1 & 0 & 0 & 0 & 1 & 0 & 1 \\
\hline \multirow{2}{*}{ P5 } & G1 & 0 & 1 & 0 & 0 & 0 & 0 & 0 & 0 \\
\cline { 2 - 10 } & G2 & 0 & 1 & 0 & 1 & 0 & 1 & 0 & 1 \\
\hline P6 & G1 & 0 & 1 & 0 & 1 & 0 & 1 & 0 & 1 \\
\hline
\end{tabular}

considération, comme l'indique l'équation (10), le pourcentage des éléments exceptionnels et le taux d'utilisation des machines.

$$
E G 1=\alpha M U+(1-\alpha)\left(1-\frac{N E}{m p-\sum_{k=1}^{Q} m_{k} p_{k}}\right)
$$

avec :

$\alpha$ un facteur d'importance relative, (généralement on prend $\alpha=0,5)$. $m p$ est la taille de la matrice d'incidence.

La deuxième définition a été proposée par [30]. Elle tient compte des charges des machines dans les cellules en terme de nombres $N E, E E$ et $P E$.

$$
E G 2=\frac{N E-E E}{N E+V E}
$$

avec :

$V E$ (Void Element) le nombre des éléments nuls dans les blocs diagonaux.

\section{Exemples illustratifs}

\subsection{Cas d'un plan d'expériences factoriel}

La méthodologie de l'approche proposée est appliquée à un exemple de formation de cellules présenté par [10]. Soit un atelier disposant de 8 machines pour fabriquer 6 types de produits :

- le produit P1 peut avoir deux gammes possibles;

- le produit P3 possède trois gammes alternatives;

- le produit P5 dispose de deux gammes alternatives;

- les produits $\mathrm{P} 2, \mathrm{P} 4$ et $\mathrm{P} 6$ possèdent chacun une seule gamme.

Le tableau 1 présente la matrice d'incidence de ce problème. La présence d'un « $1 »$ dans la matrice d'incidence désigne que la gamme du produit demande la machine correspondante. Dans le cas contraire, on met $\ll 0 »$.

Le problème consiste à choisir pour chaque produit la gamme qui donnera le meilleur groupement des machines en cellules.
Tableau 2. Les résultats du plan factoriel complet.

\begin{tabular}{|c|c|c|}
\hline Exp. & Gamme $(\mathrm{P} 1, \mathrm{P} 3, \mathrm{P} 5)$ & $P C$ \\
\hline 1 & $(1,1,1)$ & 76,1 \\
\hline 2 & $(1,1,2)$ & 85,6 \\
\hline 3 & $(1,2,2)$ & 92,5 \\
\hline 4 & $(1,3,2)$ & 85,6 \\
\hline 5 & $(1,2,1)$ & 83,9 \\
\hline 6 & $(1,3,1)$ & 76,1 \\
\hline 7 & $(2,1,1)$ & 73,3 \\
\hline 8 & $(2,1,2)$ & 80,2 \\
\hline 9 & $(2,2,1)$ & 82,1 \\
\hline 10 & $(2,3,1)$ & 69,1 \\
\hline 11 & $(2,3,2)$ & 78,4 \\
\hline 12 & $(2,2,2)$ & 91,6 \\
\hline
\end{tabular}

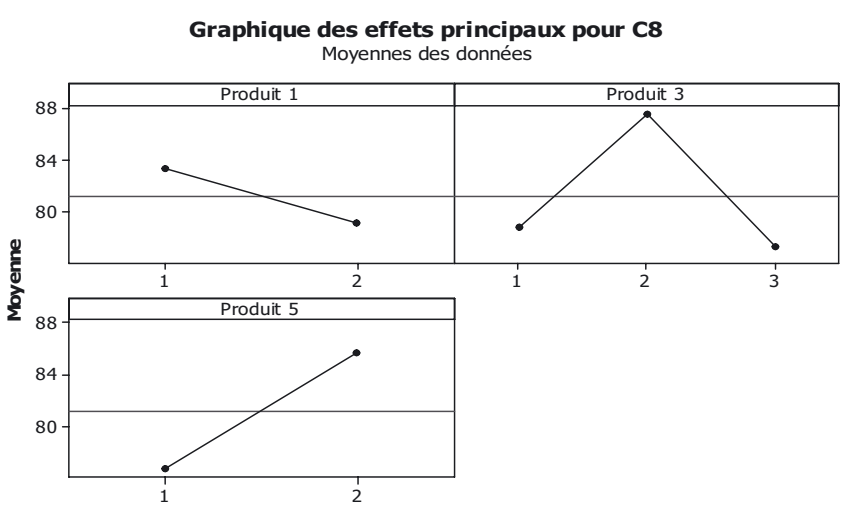

Fig. 4. Graphique des effets moyens.

\subsubsection{Phase 1 : Plan d'expériences}

En appliquant la démarche de la figure 1, l'étude du plan complet consiste à étudier toutes les combinaisons possibles des gammes de chaque produit. Le nombre d'expériences nécessaires pour l'ensemble des combinaisons qu'on note $N$, est simplement le produit du nombre de gammes de chaque produit, c'est-à-dire $N=2 \times 3 \times 2=$ 12 expériences.

Pour chaque expérience et comme l'indique le tableau 2, on a évalué par l'intermédiaire du logiciel XLSTAT (version 7.5.3) l'indice $P C$. Pour faciliter l'interprétation des résultats du plan d'expériences, nous avons utilisé le logiciel MINITAB (version 14). La figure 4 représente l'ensemble des effets qui permet de visualiser l'effet des facteurs (produit 1, produit 3 et produit 5). La figure 5 représente l'ensemble des interactions entre les différents produits.

On peut constater à partir des figures 4 et 5 qu'il faut tenir compte des seuls effets des produits P1 et P3, c'est-à-dire retenir la gamme G1 pour le facteur P1 et la gamme G2 pour le produit P3. D'après la figure 5, le produit P5 n'est pas lié par une interaction (les segments sont parallèles), il suffit donc de retenir la gamme de ce produit qui donne le maximum de PC, c'est-à-dire la gamme G2. En plus, le produit P5 ne doit pas être fabriqué dans la 


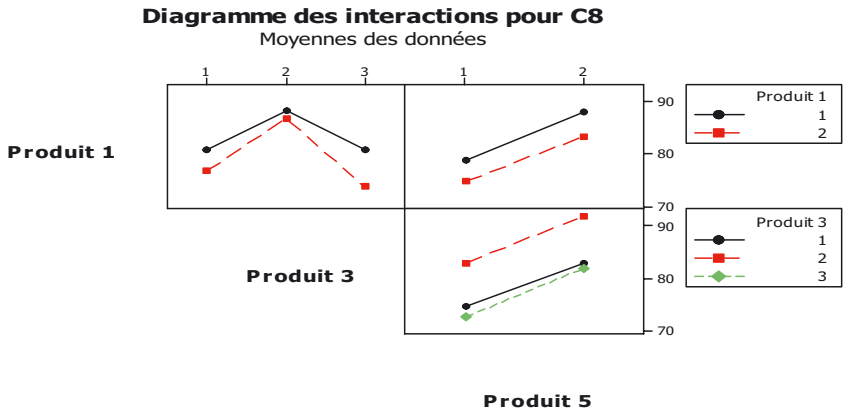

Fig. 5. Graphe des interactions.

même cellule que les produits $\mathrm{P} 1$ et $\mathrm{P} 3$ car il ne possède aucune interaction avec les produits $\mathrm{P} 1$ et P3.

La figure 4 montre que le produit P1 possède le minimum d'effet moyen. Par conséquence, la solution finale est peu sensible à un changement de la gamme préférentielle du produit P1. En plus, la solution optimale est plus sensible aux changements de la gamme de traitement du produit P5 en la comparant au produit P3. Nous voyons les intérêts supplémentaires de l'utilisation de la méthode des plans d'expérience pour la conception des systèmes de production cellulaires.

Cette première phase nous a conduit à choisir la gamme G1 pour le produit P1, la gamme G2 pour le produit P3 et la gamme G2 pour le produit P5. Cette configuration constitue la configuration optimale.

Il est à noter que cette solution (G1, G2, G2) correspond aussi à la valeur maximale de $P C$ présentée dans le tableau $2(P C=92,5 \%)$.

\subsubsection{Phase 2 : Formation des cellules}

La deuxième phase de l'approche proposée consiste à la résolution du problème classique de formation des cellules. Cette approche se base sur une matrice d'incidence binaire (Produits-Machines) avec une seule gamme par produit. Dans ce travail, nous avons utilisé l'approche FCC. La figure 6 présente le plan principal de la troisième configuration retenue à la fin de la première phase.

En tenant compte des règles d'analyse du plan principal, que nous avons présenté dans la section 2.1.2, on affirme simplement que l'atelier doit comporter deux cellules : une première cellule comprenant les machines $M_{1}$, $\mathrm{M}_{3}, \mathrm{M}_{5}$ et $\mathrm{M}_{7}$ qui traitent les produits P1, P2 et P3 et une deuxième cellule comprenant les machines $\mathrm{M}_{2}, \mathrm{M}_{4}, \mathrm{M}_{6}$ et $\mathrm{M}_{8}$ qui traitent les produits P4, P5 et P6. La solution finale est présentée dans le tableau 3.

La méthode FCC présente plusieurs points forts. L'avantage réside dans la simplicité, la rapidité de résolution et la considération du nombre de cellules à former comme une variable dépendante ou indépendante. En plus, elle est accessible gratuitement pour les industriels à travers la disponibilité de plusieurs logiciels d'analyses de données dans le marché comme SPSS, SPAD, S+, XLSTAT, etc. pour la formation des cellules et pour le calcul de l'indice de performances $P C$.

\section{Plan Principal (axes C1 et C2) PC $=92,53 \%$}

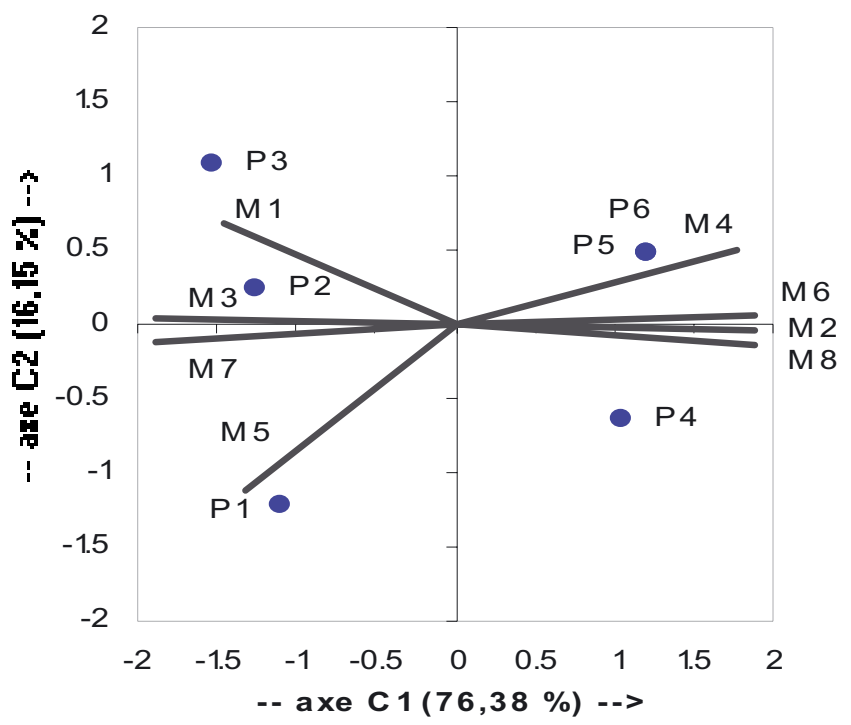

Fig. 6. Projection des machines et des produits sur le plan principal de la configuration optimale.

Tableau 3. Solution finale de l'exemple illustratif.

\begin{tabular}{|c|c|c|c|c|c|c|c|c|c|}
\hline Produit & Gamme & $\mathrm{M}_{1}$ & $\mathrm{M}_{5}$ & $\mathrm{M}_{3}$ & $\mathrm{M}_{7}$ & $\mathrm{M}_{2}$ & $\mathrm{M}_{4}$ & $\mathrm{M}_{6}$ & $\mathrm{M}_{8}$ \\
\hline 1 & 1 & 0 & 1 & 1 & 1 & \multirow{3}{*}{\multicolumn{4}{|c|}{1}} \\
\hline 2 & 1 & 1 & 0 & 1 & 1 & & & & \\
\hline 3 & 2 & 1 & 0 & 1 & 1 & & & & \\
\hline 4 & 1 & & & & & 1 & 0 & 1 & 1 \\
\hline 5 & 2 & & & & & 1 & 1 & 1 & 1 \\
\hline 6 & 1 & & & & & 1 & 1 & 1 & 1 \\
\hline
\end{tabular}

\subsubsection{Justification de l'indice $P C$}

L'objectif de ce paragraphe est de justifier la proposition de $P C$ comme un nouvel indice de performance d'une méthode de FC. Pour cela, nous avons appliqué la méthode FCC pour chaque configuration du tableau 2. Toutes les solutions finales sont présentées dans la figure 7. À l'aide de ces solutions, nous avons calculé pour chaque configuration les indices de performances : $P E$, $M U, E G 1$ et $E G 2$ à l'aide des équations (8), (9), (10) et (11).

La figure 8 présente les résultats des différents indices de performances pour les 12 configurations. Nous remarquons bien que tous ces indices évoluent pratiquement de la même manière et ils confirment que la troisième configuration est la solution optimale. En effet, cette configuration donne le maximum de MU, EG1, EG2 et $P C$ et le minimum de $P E$. On remarque aussi, la forte corrélation des différents indices de performance proposés en littérature avec l'indice $P C$ que nous avons proposé.

En plus, l'indice $P C$ n'exige pas la confection des différentes cellules pour être calculé, comme le cas des 


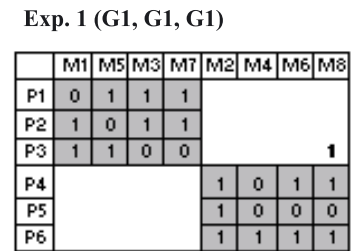

Exp. $5(G 1$, G2, G1)

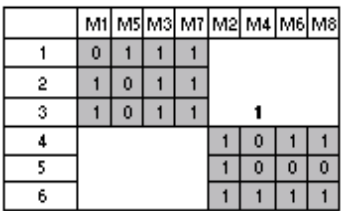

Exp. 9 (G2, G2, G1)

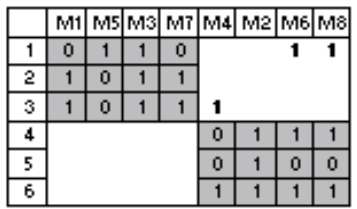

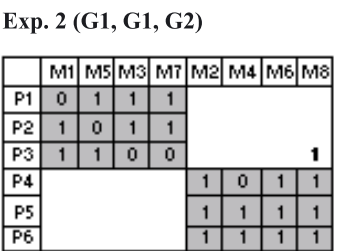

Exp. $6(\mathrm{G} 1, \mathrm{G} 3, \mathrm{G} 2)$

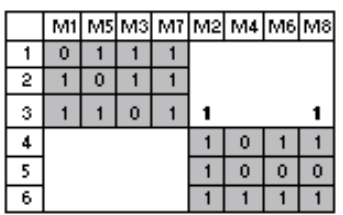

Exp. $10(G 2$, G3, G1)

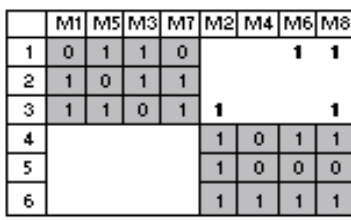

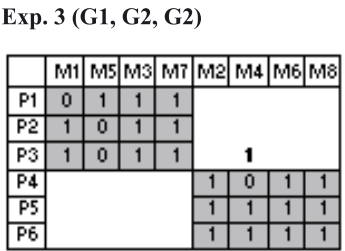

Exp. 7 (G2, G1, G1)

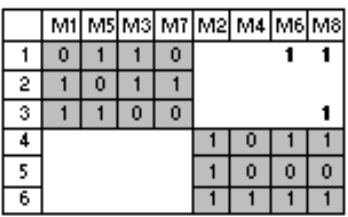

Exp. 11 (G2, G3, G2)

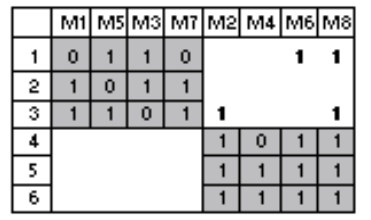

Exp. 4 (G1, G3, G2)

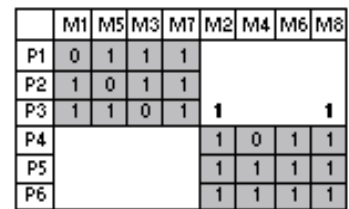

Exp. 8 (G1, G1, G2)

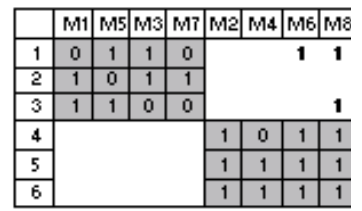

Exp. 12 (G2, G2, G2)

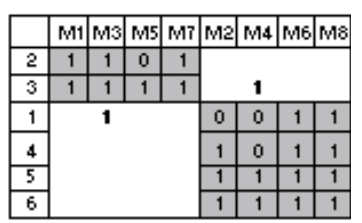

Fig. 7. Les solutions finales de toutes les configurations possibles.

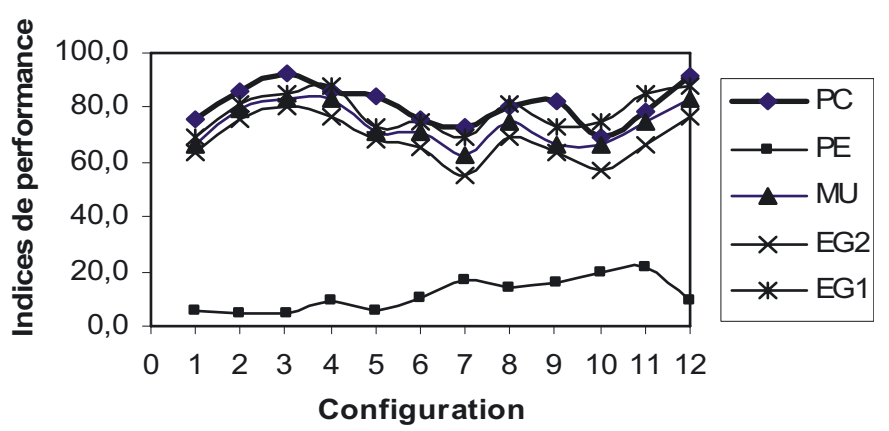

Fig. 8. Comparaison de $P C$ aux indices de performances : $P E, M U, E G 1$ et $E G 2$.

autres indices de performances qui nécessitent d'avoir toutes les solutions de la figure 7 . Il est déterminé d'une manière simple et automatique à travers la multitude des logiciels d'analyses de données aisément disponibles.

\subsection{Cas d'un plan d'expériences fractionnaire}

\subsubsection{Phase 1 : plan d'expériences}

Le problème proposé par Nagi [5] est l'un des problèmes les plus cités en littérature tels que $[6,11,16,31]$, etc. Ce problème est de grande taille, en effet, il comporte 20 machines à grouper en cinq cellules pour la production de 20 produits décrits par $51 \mathrm{~g}$. Seuls les produits P17, P18, P19 et P20 ont une seule gamme possible. Par contre les produits P5, P7, P9, P11, P12, P13, P14, P15 et $\mathrm{P} 16$ pouvant être chacun réalisé par trois gammes alternatives et les autres produits par deux gammes alternatives. Ceci engendre donc 9 facteurs à 3 niveaux et 7 facteurs
Tableau 4. Résultats des différents indices de performances.

\begin{tabular}{|c|c|c|c|c|c|c|c|c|}
\hline Exp. & $P C$ & $E E$ & $V E$ & $N E$ & $P E$ & $M U$ & $E G$ & $E G 2$ \\
\hline 1 & 76,1 & 1 & 8 & 17 & 5,9 & 66,7 & 68,8 & 64,0 \\
\hline 2 & 85,6 & 1 & 5 & 20 & 5,0 & 79,2 & 81,3 & 76,0 \\
\hline 3 & 92,5 & 1 & 4 & 21 & 4,8 & 83,3 & 85,4 & 80,0 \\
\hline 4 & 85,6 & 2 & 4 & 22 & 9,1 & 83,3 & 87,5 & 76,9 \\
\hline 5 & 83,9 & 1 & 7 & 18 & 5,6 & 70,8 & 72,9 & 68,0 \\
\hline 6 & 76,1 & 2 & 7 & 19 & 10,5 & 70,8 & 75,0 & 65,4 \\
\hline 7 & 73,3 & 3 & 9 & 18 & 16,7 & 62,5 & 68,8 & 55,6 \\
\hline 8 & 80,2 & 3 & 5 & 21 & 14,3 & 75,0 & 81,3 & 69,2 \\
\hline 9 & 82,1 & 3 & 8 & 19 & 15,8 & 66,7 & 72,9 & 59,3 \\
\hline 10 & 69,1 & 4 & 8 & 20 & 20,0 & 66,7 & 75,0 & 57,1 \\
\hline 11 & 78,4 & 4 & 5 & 23 & 17,4 & 79,2 & 87,5 & 67,9 \\
\hline 12 & 91,6 & 2 & 4 & 22 & 9,1 & 83,3 & 87,5 & 76,9 \\
\hline
\end{tabular}

à 2 niveaux. La matrice d'incidence relative au problème de Nagi est présentée dans le tableau 5.

De ce fait, le nombre minimum de ddl du modèle est 25. La table $\mathrm{L}_{36}\left(3^{9} \times 2^{7}\right)$ ayant un nombre de ddl suffisant a été sélectionnée pour appliquer l'approche proposée. Nous ne recherchons pas un modèle expliquant la liaison entre les produits, nous recherchons simplement à sélectionner la meilleure gamme de chaque produit de telle sorte que $P C$ soit maximum. Par conséquent, nous choisirons de ne pas étudier les interactions et de nous limiter aux 16 colonnes présentées dans le tableau 6 .

Le graphe des effets principaux relatif au plan d'expériences du tableau 6 est présenté dans la figure 9. À partir de cette figure, nous pouvons conclure d'une part, que les produits P5 et P8 sont les plus significatifs. Ces produits auront la plus grande influence sur la formation 
Tableau 5. La matrice d'incidence $(20 \times 20 \times 51)$ du problème de Nagi.

\begin{tabular}{|c|c|c|c|c|c|c|c|c|c|c|c|c|c|c|c|c|c|c|c|c|c|c|}
\hline \begin{tabular}{|l|} 
Ligne \\
\end{tabular} & \begin{tabular}{|l|} 
Produits \\
\end{tabular} & Gammes & $\mathrm{M}_{1}$ & $\mathrm{M}_{2}$ & $\mathrm{M}_{3}$ & $\mathrm{M}_{4}$ & $\mathrm{M}_{5}$ & $\mathrm{M}_{6}$ & $\mathrm{M}_{7}$ & $\mathrm{M}_{8}$ & $\mathrm{M}_{9}$ & $\mathrm{M}_{10}$ & $\mathrm{M}_{11}$ & $\mathrm{M}_{12}$ & $\mathrm{M}_{13}$ & $\mathrm{M}_{14}$ & $\mathrm{M}_{15}$ & $\mathrm{M}_{16}$ & $\mathrm{M}_{17}$ & $\mathrm{M}_{18}$ & $\mathrm{M}_{19}$ & $\mathrm{M}_{20}$ \\
\hline 1 & $\mathrm{P} 1$ & 1 & 0 & 0 & 0 & 0 & 0 & 1 & 0 & 0 & 1 & 0 & 0 & 1 & 0 & 0 & 0 & 0 & 0 & 0 & 0 & 0 \\
\hline 2 & & 2 & 0 & 0 & 0 & 0 & 0 & 0 & 1 & 0 & 1 & 0 & 0 & 1 & 0 & 0 & 0 & 0 & 0 & 0 & 0 & 0 \\
\hline 3 & $\mathrm{P} 2$ & 1 & 1 & 0 & 0 & 0 & 0 & 1 & 0 & 0 & 0 & 0 & 0 & 1 & 0 & 0 & 0 & 0 & 0 & 0 & 0 & 0 \\
\hline 4 & & 2 & 1 & 0 & 0 & 0 & 0 & 0 & 1 & 0 & 0 & 0 & 0 & 1 & 0 & 0 & 0 & 0 & 0 & 0 & 0 & 0 \\
\hline 5 & P3 & 1 & 1 & 0 & 0 & 0 & 0 & 1 & 0 & 0 & 1 & 0 & 0 & 1 & 0 & 0 & 0 & 0 & 0 & 0 & 0 & 0 \\
\hline 6 & & 2 & 1 & 0 & 0 & 0 & 0 & 0 & 1 & 0 & 1 & 0 & 0 & 1 & 0 & 0 & 0 & 0 & 0 & 0 & 0 & 0 \\
\hline 7 & $\mathrm{P} 4$ & 1 & 1 & 0 & 0 & 0 & 0 & 1 & 0 & 0 & 0 & 0 & 0 & 1 & 0 & 0 & 0 & 0 & 0 & 0 & 0 & 0 \\
\hline 8 & & 2 & 1 & 0 & 0 & 0 & 0 & 0 & 1 & 0 & 0 & 0 & 0 & 1 & 0 & 0 & 0 & 0 & 0 & 0 & 0 & 0 \\
\hline 9 & $\mathrm{P} 5$ & 1 & 1 & 0 & 0 & 0 & 0 & 0 & 0 & 0 & 1 & 0 & 0 & 1 & 0 & 0 & 0 & 0 & 0 & 0 & 1 & 0 \\
\hline 10 & & 2 & 1 & 0 & 0 & 0 & 0 & 0 & 0 & 0 & 1 & 0 & 0 & 1 & 0 & 0 & 0 & 0 & 0 & 1 & 0 & 0 \\
\hline 11 & & 3 & 1 & 0 & 0 & 0 & 0 & 0 & 0 & 0 & 1 & 0 & 0 & 1 & 0 & 0 & 0 & 0 & 0 & 0 & 0 & 1 \\
\hline 12 & $\mathrm{P} 6$ & 1 & 0 & 1 & 0 & 0 & 1 & 1 & 0 & 0 & 0 & 0 & 0 & 0 & 0 & 0 & 0 & 0 & 0 & 0 & 0 & 0 \\
\hline 13 & & 2 & 0 & 1 & 0 & 0 & 1 & 0 & 1 & 0 & 0 & 0 & 0 & 0 & 0 & 0 & 0 & 0 & 0 & 0 & 0 & 0 \\
\hline 15 & $\mathrm{P} 7$ & 1 & 0 & 0 & 0 & 0 & 1 & 0 & 1 & 0 & 0 & 0 & 0 & 0 & 0 & 0 & 0 & 1 & 0 & 1 & 0 & 0 \\
\hline 17 & & 2 & 0 & 0 & 0 & 0 & 1 & 1 & 0 & 0 & 0 & 0 & 0 & 0 & 0 & 0 & 0 & 1 & 0 & 0 & 1 & 0 \\
\hline 19 & & 3 & 0 & 0 & 0 & 0 & 1 & 1 & 0 & 0 & 0 & 0 & 0 & 0 & 0 & 0 & 0 & 1 & 0 & 0 & 0 & 1 \\
\hline 20 & $\mathrm{P} 8$ & 1 & 0 & 1 & 0 & 0 & 0 & 0 & 1 & 0 & 0 & 0 & 0 & 0 & 0 & 0 & 0 & 1 & 0 & 0 & 0 & 0 \\
\hline 21 & & 2 & 0 & 1 & 0 & 0 & 0 & 1 & 0 & 0 & 0 & 0 & 0 & 0 & 0 & 0 & 0 & 1 & 0 & 0 & 0 & 0 \\
\hline 23 & P9 & 1 & 0 & 1 & 0 & 0 & 0 & 1 & 0 & 0 & 0 & 0 & 0 & 0 & 0 & 0 & 0 & 1 & 0 & 0 & 1 & 0 \\
\hline 25 & & 2 & 0 & 1 & 0 & 0 & 0 & 1 & 0 & 0 & 0 & 0 & 0 & 0 & 0 & 0 & 0 & 1 & 0 & 1 & 0 & 0 \\
\hline 27 & & 3 & 0 & 1 & 0 & 0 & 0 & 1 & 0 & 0 & 0 & 0 & 0 & 0 & 0 & 0 & 0 & 1 & 0 & 0 & 0 & 1 \\
\hline 28 & $\mathrm{P} 10$ & 1 & 0 & 1 & 0 & 0 & 1 & 1 & 0 & 0 & 0 & 0 & 0 & 0 & 0 & 0 & 0 & 1 & 0 & 0 & 0 & 0 \\
\hline 29 & & 2 & 0 & 1 & 0 & 0 & 1 & 0 & 1 & 0 & 0 & 0 & 0 & 0 & 0 & 0 & 0 & 1 & 0 & 0 & 0 & 0 \\
\hline 30 & P11 & 1 & 0 & 0 & 1 & 0 & 0 & 0 & 0 & 1 & 0 & 0 & 1 & 0 & 0 & 0 & 0 & 0 & 0 & 1 & 0 & 0 \\
\hline 31 & & 2 & 0 & 0 & 1 & 0 & 0 & 0 & 0 & 1 & 0 & 0 & 1 & 0 & 0 & 0 & 0 & 0 & 0 & 0 & 1 & 0 \\
\hline 32 & & 3 & 0 & 0 & 1 & 0 & 0 & 0 & 0 & 1 & 0 & 0 & 1 & 0 & 0 & 0 & 0 & 0 & 0 & 0 & 0 & 1 \\
\hline 33 & $\mathrm{P} 12$ & 1 & 0 & 0 & 1 & 0 & 0 & 0 & 0 & 1 & 0 & 0 & 0 & 0 & 0 & 0 & 0 & 0 & 0 & 1 & 0 & 0 \\
\hline 34 & & 2 & 0 & 0 & 1 & 0 & 0 & 0 & 0 & 1 & 0 & 0 & 0 & 0 & 0 & 0 & 0 & 0 & 0 & 0 & 1 & 0 \\
\hline 35 & & 3 & 0 & 0 & 1 & 0 & 0 & 0 & 0 & 1 & 0 & 0 & 0 & 0 & 0 & 0 & 0 & 0 & 0 & 0 & 0 & 1 \\
\hline 36 & $\mathrm{P} 13$ & 1 & 0 & 0 & 1 & 0 & 0 & 0 & 0 & 1 & 0 & 0 & 1 & 0 & 0 & 0 & 0 & 0 & 0 & 1 & 0 & 0 \\
\hline 37 & & 2 & 0 & 0 & 1 & 0 & 0 & 0 & 0 & 1 & 0 & 0 & 1 & 0 & 0 & 0 & 0 & 0 & 0 & 0 & 1 & 0 \\
\hline 38 & & 3 & 0 & 0 & 1 & 0 & 0 & 0 & 0 & 1 & 0 & 0 & 1 & 0 & 0 & 0 & 0 & 0 & 0 & 0 & 0 & 1 \\
\hline 39 & $\mathrm{P} 14$ & 1 & 0 & 0 & 0 & 0 & 0 & 0 & 0 & 0 & 0 & 1 & 0 & 0 & 0 & 1 & 0 & 0 & 1 & 1 & 0 & 0 \\
\hline 40 & & 2 & 0 & 0 & 0 & 0 & 0 & 0 & 0 & 0 & 0 & 1 & 0 & 0 & 0 & 1 & 0 & 0 & 1 & 0 & 1 & 0 \\
\hline 41 & & 3 & 0 & 0 & 0 & 0 & 0 & 0 & 0 & 0 & 0 & 1 & 0 & 0 & 0 & 1 & 0 & 0 & 1 & 0 & 0 & 1 \\
\hline 42 & $\mathrm{P} 15$ & 1 & 0 & 0 & 0 & 0 & 0 & 0 & 0 & 0 & 0 & 1 & 0 & 0 & 0 & 0 & 0 & 0 & 1 & 1 & 0 & 0 \\
\hline 43 & & 2 & 0 & 0 & 0 & 0 & 0 & 0 & 0 & 0 & 0 & 1 & 0 & 0 & 0 & 0 & 0 & 0 & 1 & 0 & 1 & 0 \\
\hline 44 & & 3 & 0 & 0 & 0 & 0 & 0 & 0 & 0 & 0 & 0 & 1 & 0 & 0 & 0 & 0 & 0 & 0 & 1 & 0 & 0 & 1 \\
\hline 45 & $\mathrm{P} 16$ & 1 & 0 & 0 & 0 & 0 & 0 & 0 & 0 & 0 & 0 & 1 & 0 & 0 & 0 & 1 & 0 & 0 & 0 & 1 & 0 & 0 \\
\hline 46 & & 2 & 0 & 0 & 0 & 0 & 0 & 0 & 0 & 0 & 0 & 1 & 0 & 0 & 0 & 1 & 0 & 0 & 0 & 0 & 1 & 0 \\
\hline 47 & & 3 & 0 & 0 & 0 & 0 & 0 & 0 & 0 & 0 & 0 & 1 & 0 & 0 & 0 & 1 & 0 & 0 & 0 & 0 & 0 & 1 \\
\hline 48 & $\mathrm{P} 17$ & 1 & 0 & 0 & 0 & 0 & 0 & 0 & 0 & 0 & 0 & 1 & 0 & 0 & 0 & 1 & 0 & 0 & 0 & 0 & 0 & 0 \\
\hline 49 & P18 & 1 & 0 & 0 & 0 & 1 & 0 & 0 & 0 & 0 & 0 & 0 & 0 & 0 & 1 & 0 & 1 & 0 & 0 & 0 & 0 & 0 \\
\hline 50 & P19 & 1 & 0 & 0 & 0 & 1 & 0 & 0 & 0 & 0 & 0 & 0 & 0 & 0 & 1 & 0 & 1 & 0 & 0 & 0 & 0 & 0 \\
\hline 51 & P20 & 1 & 0 & 0 & 0 & 1 & 0 & 0 & 0 & 0 & 0 & 0 & 0 & 0 & 0 & 0 & 1 & 0 & 0 & 0 & 0 & 0 \\
\hline
\end{tabular}


Tableau 6. Table $\mathrm{L}_{36}\left(3^{9} \times 2^{7}\right)$ relative au problème de Nagi.

\begin{tabular}{|c|c|c|c|c|c|c|c|c|c|c|c|c|c|c|c|c|c|}
\hline Expérience & $\mathrm{P} 1$ & $\mathrm{P} 2$ & P3 & $\mathrm{P} 4$ & $\mathrm{P} 5$ & $\mathrm{P} 6$ & $\mathrm{P} 7$ & $\mathrm{P} 8$ & $\mathrm{P} 9$ & $\mathrm{P} 10$ & $\mathrm{P} 11$ & $\mathrm{P} 12$ & $\mathrm{P} 13$ & $\mathrm{P} 14$ & $\mathrm{P} 15$ & $\mathrm{P} 16$ & $P C$ \\
\hline 1 & 1 & 1 & 1 & 1 & 1 & 1 & 1 & 2 & 1 & 2 & 1 & 2 & 2 & 1 & 2 & 3 & 36,9 \\
\hline 2 & 1 & 1 & 1 & 1 & 2 & 1 & 2 & 2 & 2 & 2 & 2 & 3 & 3 & 3 & 3 & 2 & 37,8 \\
\hline 3 & 1 & 1 & 1 & 1 & 3 & 1 & 3 & 2 & 3 & 2 & 3 & 1 & 1 & 2 & 1 & 1 & 37,9 \\
\hline 4 & 1 & 1 & 2 & 2 & 1 & 2 & 1 & 1 & 2 & 1 & 3 & 1 & 2 & 3 & 3 & 2 & 36,5 \\
\hline 5 & 1 & 1 & 2 & 2 & 2 & 2 & 2 & 1 & 3 & 1 & 1 & 2 & 3 & 2 & 1 & 1 & 35,8 \\
\hline 6 & 1 & 1 & 2 & 2 & 3 & 2 & 3 & 1 & 1 & 1 & 2 & 3 & 1 & 1 & 2 & 3 & 36,5 \\
\hline 7 & 1 & 2 & 1 & 2 & 1 & 2 & 1 & 1 & 3 & 2 & 2 & 1 & 3 & 1 & 3 & 1 & 36,2 \\
\hline 8 & 1 & 2 & 1 & 2 & 2 & 2 & 2 & 1 & 1 & 2 & 3 & 2 & 1 & 3 & 1 & 3 & 36,5 \\
\hline 9 & 1 & 2 & 1 & 2 & 3 & 2 & 3 & 1 & 2 & 2 & 1 & 3 & 2 & 2 & 2 & 2 & 36,4 \\
\hline 10 & 1 & 2 & 2 & 1 & 1 & 2 & 2 & 2 & 3 & 1 & 1 & 3 & 2 & 2 & 3 & 3 & 37,6 \\
\hline 11 & 1 & 2 & 2 & 1 & 2 & 2 & 3 & 2 & 1 & 1 & 2 & 1 & 3 & 1 & 1 & 2 & 36,4 \\
\hline 12 & 1 & 2 & 2 & 1 & 3 & 2 & 1 & 2 & 2 & 1 & 3 & 2 & 1 & 3 & 2 & 1 & 36,2 \\
\hline 13 & 1 & 2 & 2 & 2 & 1 & 1 & 2 & 2 & 3 & 2 & 2 & 1 & 1 & 3 & 2 & 3 & 37,9 \\
\hline 14 & 1 & 2 & 2 & 2 & 2 & 1 & 3 & 2 & 1 & 2 & 3 & 2 & 2 & 2 & 3 & 2 & 37,5 \\
\hline 15 & 1 & 2 & 2 & 2 & 3 & 1 & 1 & 2 & 2 & 2 & 1 & 3 & 3 & 1 & 1 & 1 & 37,3 \\
\hline 16 & 2 & 1 & 2 & 2 & 1 & 1 & 2 & 1 & 1 & 2 & 3 & 3 & 3 & 2 & 2 & 1 & 39,7 \\
\hline 17 & 2 & 1 & 2 & 2 & 2 & 1 & 3 & 1 & 2 & 2 & 1 & 1 & 1 & 1 & 3 & 3 & 38,3 \\
\hline 18 & 2 & 1 & 2 & 2 & 3 & 1 & 1 & 1 & 3 & 2 & 2 & 2 & 2 & 3 & 1 & 2 & 39,2 \\
\hline 19 & 2 & 1 & 2 & 1 & 1 & 2 & 2 & 2 & 2 & 2 & 3 & 3 & 1 & 1 & 1 & 2 & 37,3 \\
\hline 20 & 2 & 1 & 2 & 1 & 2 & 2 & 3 & 2 & 3 & 2 & 1 & 1 & 2 & 3 & 2 & 1 & 37,4 \\
\hline 21 & 2 & 1 & 2 & 1 & 3 & 2 & 1 & 2 & 1 & 2 & 2 & 2 & 3 & 2 & 3 & 3 & 36,3 \\
\hline 22 & 2 & 1 & 1 & 2 & 1 & 2 & 3 & 2 & 2 & 1 & 1 & 2 & 3 & 3 & 1 & 3 & 36,0 \\
\hline 23 & 2 & 1 & 1 & 2 & 2 & 2 & 1 & 2 & 3 & 1 & 2 & 3 & 1 & 2 & 2 & 2 & 36,0 \\
\hline 24 & 2 & 1 & 1 & 2 & 3 & 2 & 2 & 2 & 1 & 1 & 3 & 1 & 2 & 1 & 3 & 1 & 36,1 \\
\hline 25 & 2 & 2 & 2 & 1 & 1 & 1 & 3 & 1 & 2 & 1 & 2 & 2 & 1 & 2 & 3 & 1 & 37,5 \\
\hline 26 & 2 & 2 & 2 & 1 & 2 & 1 & 1 & 1 & 3 & 1 & 3 & 3 & 2 & 1 & 1 & 3 & 37,0 \\
\hline 27 & 2 & 2 & 2 & 1 & 3 & 1 & 2 & 1 & 1 & 1 & 1 & 1 & 3 & 3 & 2 & 2 & 38,8 \\
\hline 28 & 2 & 2 & 1 & 2 & 1 & 1 & 3 & 2 & 3 & 1 & 3 & 2 & 3 & 1 & 2 & 2 & 38,8 \\
\hline 29 & 2 & 2 & 1 & 2 & 2 & 1 & 1 & 2 & 1 & 1 & 1 & 3 & 1 & 3 & 3 & 1 & 37,5 \\
\hline 30 & 2 & 2 & 1 & 2 & 3 & 1 & 2 & 2 & 2 & 1 & 2 & 1 & 2 & 2 & 1 & 3 & 38,7 \\
\hline 31 & 2 & 2 & 1 & 1 & 1 & 2 & 3 & 1 & 1 & 2 & 2 & 3 & 2 & 3 & 1 & 1 & 35,9 \\
\hline 32 & 2 & 2 & 1 & 1 & 2 & 2 & 1 & 1 & 2 & 2 & 3 & 1 & 3 & 2 & 2 & 3 & 37,4 \\
\hline 33 & 2 & 2 & 1 & 1 & 3 & 2 & 2 & 1 & 3 & 2 & 1 & 2 & 1 & 1 & 3 & 2 & 37,4 \\
\hline 34 & 2 & 2 & 2 & 2 & 3 & 1 & 3 & 2 & 3 & 1 & 2 & 2 & 2 & 2 & 1 & 2 & 42,7 \\
\hline 35 & 2 & 2 & 2 & 2 & 1 & 1 & 3 & 2 & 3 & 1 & 1 & 1 & 1 & 1 & 2 & 1 & 43,9 \\
\hline 36 & 2 & 2 & 2 & 2 & 2 & 1 & 2 & 2 & 1 & 1 & 1 & 1 & 1 & 3 & 3 & 3 & 42,4 \\
\hline
\end{tabular}

des cellules. D'autre part en choisissant les points les plus hauts, la configuration optimale consiste à affecter :

- la première gamme pour chacun des produits P5, P6, $\mathrm{P} 10, \mathrm{P} 11, \mathrm{P} 12$ et $\mathrm{P} 13$;

- la deuxième gamme pour les produits P1, P2, P3, P4, $\mathrm{P} 8, \mathrm{P} 14, \mathrm{P} 15$ et $\mathrm{P} 16$;

- la troisième gamme pour les produits $\mathrm{P} 7$ et $\mathrm{P} 9$.

La solution finale est indiquée dans la deuxième et la troisième colonne du tableau 7 .

\subsubsection{Phase 2 : Formation des cellules}

En appliquant l'approche FCC tout en analysant le plan principal indiqué dans la figure 10, nous assurons simplement que l'atelier doit comporter cinq cellules comme l'indique le tableau 7.

Pour évaluer les résultats de l'approche proposée, nous avons consulté les meilleures solutions qui ont été publiées en littérature pour le problème de Nagi [6,31]. Toutes les solutions présentées engendrent les mêmes critères de performances. Une analyse comparative portant sur les gammes sélectionnées dans les différentes solutions est indiquée dans le tableau 8. La solution obtenue est différente à celles proposées par [6] et par [31].

\section{Validation}

Le tableau 9 résume quelques tests qui ont été réalisés en appliquant l'approche proposée sur des matrices 
Graphique des effets principaux relatif au problème de Nagi Moyennes des $\mathrm{PC}$

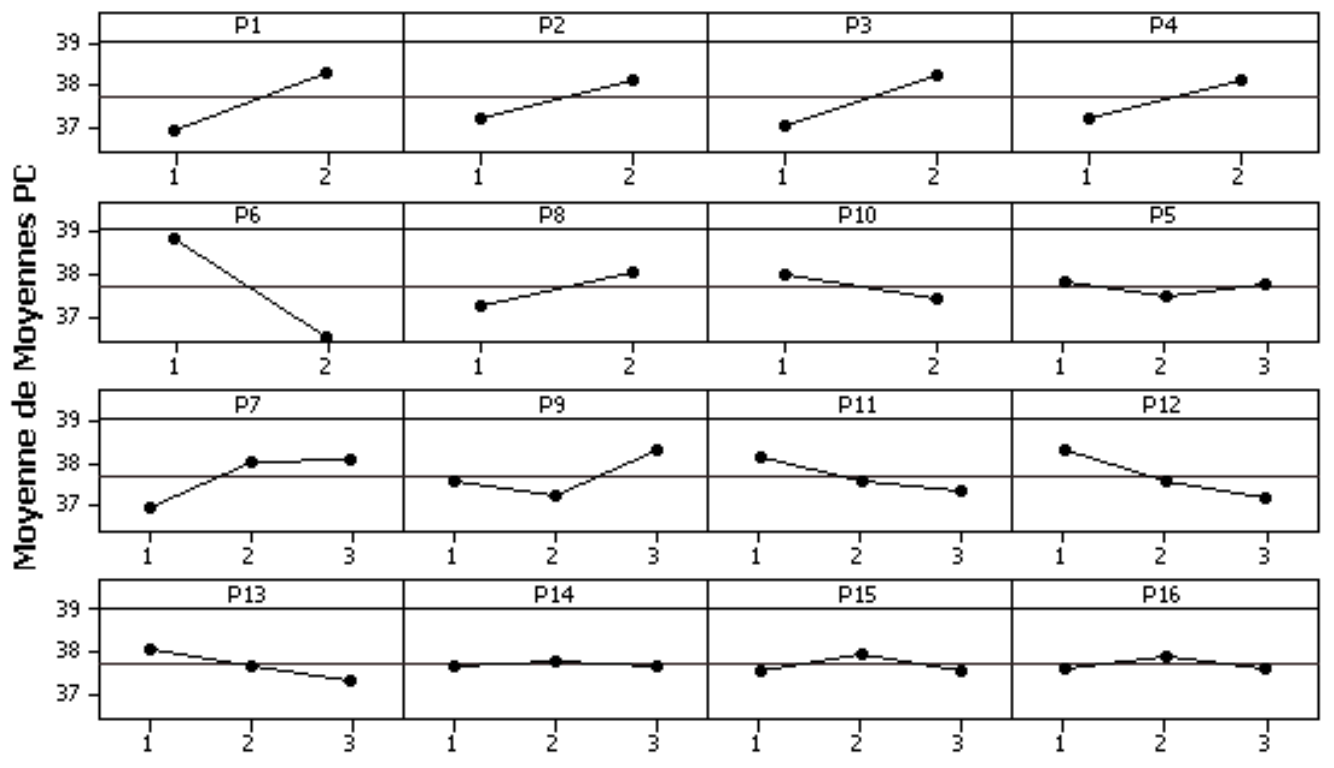

Fig. 9. Graphique des effets moyens relatifs au problème de Nagi.

Scatter Plot (axes F1 et F2) PC = 43,91 \%)

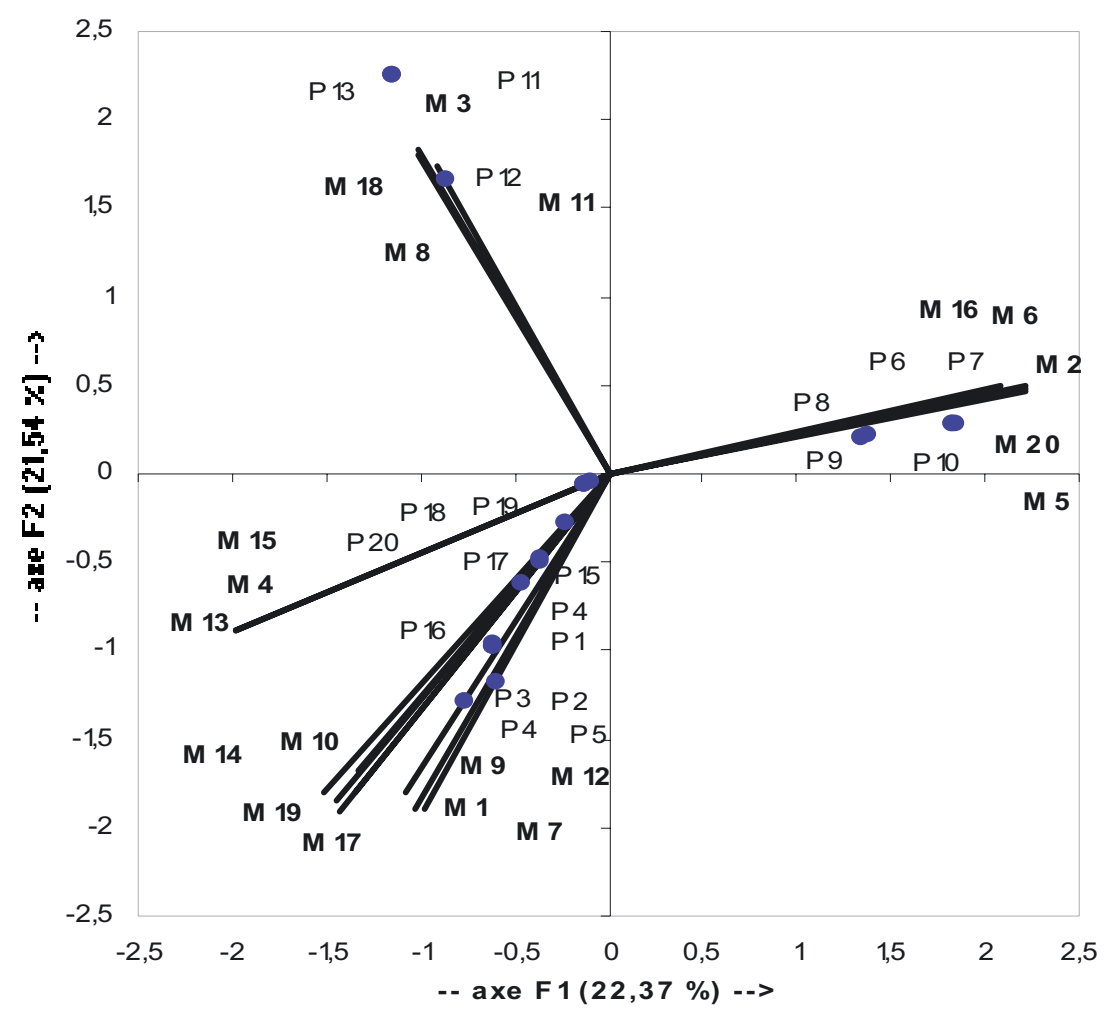

Fig. 10. Plan principal de la configuration optimale pour le problème de Nagi. 
Tableau 7. La solution finale obtenue du problème de Nagi.

\begin{tabular}{|c|c|c|c|c|c|c|c|c|c|c|c|c|c|c|c|c|c|c|c|c|c|c|}
\hline Ligne & Produits & Gammes & M2 & M5 & M6 & M16 & M20 & M10 & M14 & M17 & M19 & M3 & M8 & M11 & M18 & M1 & M7 & M9 & M12 & M4 & M13 & M15 \\
\hline 12 & P6 & 1 & 1 & 1 & 1 & 0 & 0 & & & & & & & & & & & & & & & \\
\hline 19 & $\mathrm{P} 7$ & 3 & 0 & 1 & 1 & 1 & 1 & & & & & & & & & & & & & & & \\
\hline 21 & P8 & 2 & 1 & 0 & 1 & 1 & 0 & & & & & & & & & & & & & & & \\
\hline 27 & P9 & 3 & 1 & 0 & 1 & 1 & 1 & & & & & & & & & & & & & & & \\
\hline 28 & $\mathrm{P} 10$ & 1 & 1 & 1 & 1 & 1 & 0 & & & & & & & & & & & & & & & \\
\hline 40 & P14 & 2 & & & & & & 1 & 1 & 1 & 1 & & & & & & & & & & & \\
\hline 43 & P15 & 2 & & & & & & 1 & 0 & 1 & 1 & & & & & & & & & & & \\
\hline 46 & P16 & 2 & & & & & & 1 & 1 & 0 & 1 & & & & & & & & & & & \\
\hline 48 & P17 & 1 & & & & & & 1 & 1 & 0 & 0 & & & & & & & & & & & \\
\hline 30 & P11 & 1 & & & & & & & & & & 1 & 1 & 1 & 1 & & & & & & & \\
\hline 33 & $\mathrm{P} 12$ & 1 & & & & & & & & & & 1 & 1 & 0 & 1 & & & & & & & \\
\hline 36 & P13 & 1 & & & & & & & & & & 1 & 1 & 1 & 1 & & & & & & & \\
\hline 2 & $\mathrm{P} 1$ & 2 & & & & & & & & & & & & & & 0 & 1 & 1 & 1 & & & \\
\hline 4 & P2 & 2 & & & & & & & & & & & & & & 1 & 1 & 0 & 1 & & & \\
\hline 6 & P3 & 2 & & & & & & & & & & & & & & 1 & 1 & 1 & 1 & & & \\
\hline 8 & P4 & 2 & & & & & & & & & & & & & & 1 & 1 & 0 & 1 & & & \\
\hline 9 & P5 & 1 & & & & & & & & & 1 & & & & & 1 & 0 & 1 & 1 & & & \\
\hline 49 & P18 & 1 & & & & & & & & & & & & & & & & & & 1 & 1 & 1 \\
\hline 50 & P19 & 1 & & & & & & & & & & & & & & & & & & 1 & 1 & 1 \\
\hline 51 & P20 & 1 & & & & & & & & & & & & & & & & & & 1 & 0 & 1 \\
\hline
\end{tabular}

Tableau 8. Comparaison entre la solution proposée et celles proposées en littérature.

\begin{tabular}{|c|c|c|c|c|c|c|c|c|c|c|c|c|c|c|c|c|c|c|c|c|}
\hline \multicolumn{11}{|c|}{ La solution finale proposée du problème de Nagi } \\
\hline Produit & 1 & 2 & 3 & 4 & 5 & 6 & 7 & 8 & 9 & 10 & 11 & 12 & 13 & 14 & 15 & 16 & 17 & 18 & 19 & 20 \\
\hline Gamme & 2 & 2 & 2 & 2 & 1 & 1 & 3 & 2 & 3 & 1 & 1 & 1 & 1 & 2 & 2 & 2 & 1 & 1 & 1 & 1 \\
\hline \multicolumn{110}{|c|}{ La solution du problème de Nagi proposée par [6] } \\
\hline Produit & 1 & 2 & 3 & 4 & 5 & 6 & 7 & 8 & 9 & 10 & 11 & 12 & 13 & 14 & 15 & 16 & 17 & 18 & 19 & 20 \\
\hline Gamme & 2 & 2 & 2 & 2 & 3 & 1 & 3 & 2 & 3 & 1 & 2 & 2 & 2 & 1 & 1 & 1 & 1 & 1 & 1 & 1 \\
\hline \multicolumn{110}{|c|}{ La solution du problème de Nagi proposée par [31] } \\
\hline Produit & 1 & 2 & 3 & 4 & 5 & 6 & 7 & 8 & 9 & 10 & 11 & 12 & 13 & 14 & 15 & 16 & 17 & 18 & 19 & 20 \\
\hline Gamme & 2 & 2 & 2 & 2 & 2 & 1 & 2 & 2 & 2 & 1 & 1 & 1 & 1 & 3 & 3 & 3 & 1 & 1 & 1 & 1 \\
\hline
\end{tabular}

d'incidence binaires avec gammes alternatives reconnues en littérature de taille différente. La source de ces problèmes est indiquée dans la dernière colonne de droite.

Afin d'évaluer l'approche proposée et de la comparer avec les autres méthodes, les résultats obtenus sont évalués en se basant sur des indicateurs de performance que nous avons présentés à la section 2.3. Le tableau 10 présente les résultats de l'approche proposée ainsi que les meilleurs résultats connus dans les articles récents. Ces travaux ont utilisé des techniques basées sur la programmation mathématique que ce soit avec résolution exacte comme dans [16,32] ou avec résolution approchée comme dans $[30,34]$. Chacun a affirmé qu'il a trouvé une solution meilleure que celle proposée en littérature. Les résultats de l'approche proposée sont globalement identiques à ceux présentés dans ces récents papiers en terme d'indicateurs de performance (PEE, EG1 et EG2).

\section{Conclusion}

Dans cet article, nous proposons une nouvelle approche pour la formation des cellules en présence de
Tableau 9. Les différents problèmes avec gammes alternatives.

\begin{tabular}{|c|c|c|c|}
\hline No. & Taille $(m \times p \times r)$ & $F$ & Référence \\
\hline 1 & $5 \times 7 \times 11$ & 2 & {$[2]$} \\
\hline 2 & $6 \times 10 \times 20$ & 2 & {$[32]$} \\
\hline 3 & $7 \times 14 \times 32$ & 3 & {$[33]$} \\
\hline 4 & $9 \times 6 \times 13$ & 3 & {$[34]$} \\
\hline 5 & $20 \times 20 \times 51$ & 5 & {$[5]$} \\
\hline
\end{tabular}

gammes alternatives. L'utilisation de la méthode des plans d'expériences permet, d'une part, le choix guidé expérimentalement de la gamme préférentielle pour chaque produit, d'autre part, une analyse de sensibilité de la solution finale. Par ailleurs, nous avons proposé et validé un nouvel indice de performance qui se base sur la conservation statistique des données de la matrice d'incidence. Cet indice permet une mesure précoce du degré de la mise en cellules d'un problème de formation cellulaire. De ce fait, il constitue la réponse aux différentes expériences du plan d'expériences factoriel 
Tableau 10. Les résultats de l'approche proposée.

\begin{tabular}{|c|c|c|c|c|c|c|c|c|c|}
\hline \multirow{2}{*}{ No. } & \multirow{2}{*}{ Taille } & \multirow{2}{*}{$F$} & \multicolumn{2}{|c|}{ Les résultas de l'approche proposée } & \multicolumn{4}{|c|}{ Les meilleurs résultas connu } \\
\cline { 4 - 10 } & & & $P E E$ & $E G 1$ & $E G 2$ & $P E E$ & $E G 1$ & $E G 2$ & Référence du résultat \\
\hline 1 & $4 \times 5 \times 11$ & 2 & 0 & 95,0 & 90,0 & 0,0 & 95,0 & 90,0 & {$[34]$} \\
\hline 2 & $6 \times 10 \times 20$ & 2 & 14,3 & 83,3 & 70,6 & 22,2 & 75,0 & 60,0 & {$[32]$} \\
\hline 3 & $7 \times 14 \times 32$ & 3 & 16,7 & 85,7 & 67,6 & 16,7 & 85,7 & 67,6 & {$[16]$} \\
\hline 4 & $9 \times 6 \times 13$ & 3 & 0,0 & 97,2 & 94,4 & 0,0 & 97,2 & 94,4 & {$[34]$} \\
\hline 5 & $20 \times 20 \times 51$ & 5 & 1,52 & 89,5 & 78,3 & 1,52 & 89,5 & 78,3 & {$[31]$} \\
\hline
\end{tabular}

ou fractionnaire. En plus, l'approche proposée présente plusieurs avantages tels que : sa simplicité de résolution et sa considération du nombre de cellules à former comme une variable dépendante ou indépendante.

Néanmoins, l'approche proposée possède des limites que nous sommes en cours d'étudier. En effet, le passage entre les différentes étapes de l'approche proposée, le choix du plan d'expériences et l'examen du plan principal, restent des opérations manuelles. Le développement informatique de cette approche, s'avère une perspective majeure. D'autre part, l'approche proposée ne tient pas en considération d'autres paramètres intéressants de production tel que la séquence des opérations et la capacité des machines. Une autre perspective intéressante serait dans l'intégration de ces aspects dans la matrice de similarité. Dans ce cas, seule une approche multicritère semble intéressante.

Remerciements. Nous exprimons nos remerciements aux membres du comité de rédaction de la revue "Mécanique et Industries" d'avoir accepté de juger et de valoriser ce travail. Les remarques et suggestions évoquées par les deux experts ont permis d'améliorer la qualité scientifique et la présentation de cet article.

\section{Références}

[1] J. Burbidge, Change to group technology : process organization is obsolete, Int. J. Production Research 30 (1992) 1209-1219

[2] A. Kusiak, The generalized group technology concept, Int. J. Production Research 25 (1987) 561-569

[3] Burgess, Morgan, Vollmann, Cellular manufacturing : its impact on the total factory, Int. J. Production Research 31 (1993) 2059-2077

[4] R. Rajamani, R. Singh, Y.P. Aneja, Integrated design of cellular manufacturing systems in the presence of alternative process plans, Int. J. Production Research 28 (1990) $1541-1554$

[5] R. Nagi, G. Harhalakis, J. Proth, Multiple routings and capacity considerations in Group Technology applications. Int. J. Production Research 28 (1990) 2243-2257

[6] J.A Joines, C.T. Gulberth, R.E. King, A genetic algorithm based integer program for manufacturing design, Proceeding of the International conference Flexible on Automatic integrated manufacturing, Stuttgart, Germany, 1995
[7] Y.C. Ho, C.L. Moodie, Solving cell formation problems in a manufacturing environment with flexible processing and routing capabilities, Int. J. Production Research 34 (1996) 2901-2923

[8] N. Singh, D. Rajamani, Cellular manufacturing systems : Design, Planning and control, First edition, Chapman and Hall, (éd.), New York, USA, 1996

[9] J.L. Paris, C. Caux, H. Pierreval, Les approches évolutionnistes, Chapitre 12, Traité IC2 Évaluation des performances des systèmes de production, Hermès (éd.), Paris, France, 2003

[10] F.T.S. Chan, K.W. Lau, P.L.Y. Chan, K.L. Choy, Two-stage approach for machine-part grouping and cell layout problems, Robotics and Computer Integrate Manufacturing, 2005

[11] B. Adenso-Diaz, S. Lozano, J. Racero, F. Guerrero, Machine cell formation in generalized group technology, Computers and Industrial Engineering 41 (2001) 227-240

[12] T. Gupta, Design of manufacturing cells for flexible environment considering alternative routeing, Int. J. Production Research 31 (1993) 1259-1273

[13] C. Caux, R. Bruniaux, H. Pierreval, Cell formation with alternative process plans and machine capacity constraints : A new combined approach, Int. J. Economics 64 (2000) 279-284

[14] E. Vin, P. De Lit, A. Delchambre, Une approche intégrée pour le problème de formation de cellules de production avec des routages alternatives, Conférence Internationale de Modélisation et SIMulation (MOSIM'03), Toulouse, France, 2003

[15] S. Gangan, S. Khator, A.J.G. Babu, A within-cell utilization based heuristic for designing cellular manufacturing systems, Computers and Industrial Engineering 13 (1987) $112-117$

[16] H. Hwang, P. Ree, Routes selection for the cell formation problem with alternative part process plans, Computers and Industrial engineering 30 (1996) 423-431

[17] M. Kazerooni, L.H.S. Luong, K. Abhary, A genetic algorithm based cell design considering alternative routing, Computer Integrated Manufacturing Systems 10 (1997) 93-107

[18] M. Kizil, M. Ozbayrak, A tradeoff analysis between process plan selection and cell formation in cellular manufacturing, Int. J. Advanced Manufacturing and Technology 23 (2004) 501-506

[19] B. Arvindh, S.A. Irani, Principal components analysis for evaluating the feasibility of cellular manufacturing without initial-part matrix clustering, Int. J. Production Research 32 (1994) 1909-1938

[20] Z. Albadawi, H.A. Bashir, M. Chen, A mathematical approach for the formation of manufacturing cell, Computers and Industrial Engineering 48 (2005) 3-21 
[21] W. Hachicha, F. Masmoudi, M. Haddar, Formation of machine groups and part families in cellular manufacturing systems using a correlation analysis approach, Int. J. Advanced Manufacturing Technology 36 (2008) 11571169

[22] W. Hachicha, F. Masmoudi, M. Haddar, A correlation analysis approach of cell formation in cellular manufacturing system with incorporated production data, Int. J. Manufacturing Research 1 (2006) 332-353

[23] M. Pillet, Introduction aux plans d'expériences par la méthode Taguchi, édition d'organisation, $2^{\mathrm{e}}$ édition, Paris, France, 1994

[24] W.D. Kelton, Designing Simulation Experiments, Proceedings of the 1999 Winter Simulation Conference, 1999 pp. 33-38

[25] J. Wang, Formation of machine cells and part families in cellular manufacturing systems using a linear assignment algorithm, Automatica 39 (2003) 1607-1615

[26] Y. Won, K.C. Lee, Modified p-median approach for efficient GT cell formation, Computers and Industrial Engineering 46 (2004) 495-510

[27] A. Stawowy, Evolutionary strategy for manufacturing cell design, The international Journal of Management Science Omega 34 (2006) 1-18
[28] W. Hachicha, F. Masmoudi, M. Haddar, An improvement of cellular manufacturing system design using simulation analysis, Int. J. simulation modelling 6 (2007) 193-205

[29] M.P. Chandrasekharan, R. Rajagopalan, An ideal seed non-hierarchical clustering algorithm for cellular manufacturing, Int. J. Production Research 24 (1986) 451-464

[30] K.R. Kumar, M.P. Chandrasekharan, Grouping efficacy : a quantitative criterion for goodness of block diagonal forms of binary matrices in group technology, Int. J. Production Research 28 (1990) 233-243

[31] M.K. Uddin, K. Shanker, Grouping of parts and machines in presence of alternative process routes by genetic algorithm, Int. J. Production Economics 76 (2002) 219-228

[32] S. Sankaran, R. Kasilingam, An integrated approach to cell formation and part routing in Group Technology manufacturing system, Engineering Optimization 16 (1990) 235-245

[33] R. Logendran, P. Ramakrishna, C. Striskandarajah, Tabu search-based heuristics for cellular manufacturing systems in the presence of alternative process plans, Int. J. Production Research 32 (1994) 273-297

[34] A.M. Mukattash, M.B. Adil, K.K. Tahboub, Heuristic approaches for part assignment in cell formation, Computers and Industrial Engineering 42 (2002) 329-341 